\title{
Use of Suture tightening automated device COR-KNOT® for minimally invasive heart valve surgery: Our initial experience in Bangladesh
}

\author{
Md Faizus Sazzad ${ }^{1}$, Nusrat Ghafoor ${ }^{2}$, Siba Pada Roy ${ }^{3}$, Swati Munshi ${ }^{4}$, Feroza Khanam ${ }^{5}$, \\ Prasanta Kumar Chanda ${ }^{6}$, Armane Wadud ${ }^{7}$, Sirajul Islam ${ }^{8}$, Farooque Ahmed ${ }^{9}$, Masoom Siraj ${ }^{10}$, \\ Ti Lian Kah ${ }^{11}$, Theodoros Kofidis ${ }^{12}$
}

\begin{abstract}
:
Background: COR-KNOT® (LSI Solutions, New York, NY, USA) is an automated suture securing device has not been well known. We report a case series for first automated knotting device used for minimally invasive heart valve surgery in Bangladesh.

Method and Results: To overcome the challenge of knot securing via a Key-Hole surgery we have used CORKNOT $\circledast$. The newest device is capable of remotely and automatically secure sutures and simultaneously can cut and remove the excess suture tails. We covered the spectrum of heart valve surgery: There was one case of bioprosthetic aortic valve replacement, one case of mitral valve repair, one case of bioprosthetic mitral valve
\end{abstract}

replacement, one case of failed mitral valve repair with COR-KNOT® explantation followed by mechanical mitral valve replacement and one case of redo-mitral valve replacement. Average length of hospital stays was $5 \pm$ 1 days. There was one reopening, one post-operative atrial fibrillation. No wound infection and no 30 day mortality.

Conclusion: We conclude, COR-KNOT® is a safe and effective tool to reduce the duration of operation. Clinical outcome of heart valve surgery with COR-KNOT® is comparable with other methods of suture tying methods.

Keywords: COR-KNOT, Minimally invasive heart valve surgery

(Bangladesh Heart Journal 2019; 34(2): 127-131)

1. Research Associate/Cardiac Surgeon, Dept. of Surgery (CTVS), Yong Loo Lin School of Medicine National University of Singapore.

2. Associate Professor \& Consultant, Dept. of Radiology \& Imaging, Ibrahim Cardiac Hospital \& Research Institute, Dhaka, Bangladesh.

3. Assistant Professor \& Associate Consultant, Dept. of Cardiac Anaesthesiology, Ibrahim Cardiac Hospital \& Research Institute, Dhaka, Bangladesh.

4. Specialist Perfutionist, Ibrahim Cardiac Hospital \& Research Institute, Dhaka, Bangladesh.

5. Associate Professor \& Senior Consultant, Dept. of Cardiac Surgery, National Heart Foundation Hospital \& Research Institute, Dhaka, Bangladesh.

6. Assistant Professor \& Associate Consultant, Department of Cardiac Anaesthesiology, Ibrahim Cardiac Hospital \& Research Institute, Dhaka, Bangladesh.

7. Senior Consultant, Dept. of Cardiac Anaesthesiology, Ibrahim Cardiac Hospital \& Research Institute, Dhaka, Bangladesh.

8. Chief Cardiac Surgeon, Dept. of Cardiac Surgery, National Heart Foundation Hospital \& Research Institute, Dhaka, Bangladesh.

9. Head of the Department, Dept. of Cardiac Surgery, Ibrahim Cardiac Hospital \& Research Institute, Dhaka, Bangladesh.

10. Haed of Anesthesiology, National University Hospital, Singapore

11. Head of the Dept., Cardiac Thoracic and Vascular Surgery, National University Hospital, Singapore

12. Specilalist, Dept. of Radiology and Imaging, Square Hospital, Dhaka, Bangladesh.

Address of Correspondence: Md Faizus Sazzad, MBBS, MS, FCPS, FACS, Research Associate/Cardiac Surgeon, Department of Surgery (CTVS), Yong Loo Lin School of Medicine, National University of Singapore, MD-6 Building, Level-08 \#South, 14, Medical Drive, Singapore 117599, Office: +65-66011434, Fax: +65-6775 8538, e-mail: surmfs@nus.edu.sg

DOI: https://doi.org/10.3329/bhj.v34i2.44444

Copyright $\odot 2017$ Bangladesh Cardiac Society. Published by Bangladesh Cardiac Society. This is an Open Access articles published under the Creative Commons Attribution-NonCommercial 4.0 International License (CC BY-NC). This license permits use, distribution and reproduction in any medium, provided the original work is properly cited and is not used for commercial purposes. 


\section{Introduction:}

One of the basic skills of a surgeon is suturing and knot tying. To perform knot tying however, can be a tedious and time-consuming especially in minimally invasive heart valve surgery. This difficulty is largely related to the limited working space, limited degree of freedom for movement of surgical instruments. Hence, suture fixation is the main hindrance for surgeons in minimally invasive heart valve surgery ${ }^{1}$.

Usually during a heart valve repair or replacement surgery, a swing ring or band is stitched and secured around the annulus. In most of the commercially available prosthetic heart valve, there is an incorporated swing ring. Commonly, surgeons takes 12 to 15 pledgeted braided interrupted mattress stitches around the ring of the valve and then fasten the sutures by hand-tying knots. Especially in minimally invasive heart surgery, where access is difficult, surgeons hand cannot reach the operation site. A knot-pusher instrument is used if the operative field is beyond the reach of the surgeon's hand, which requires more time to secure the knots.

COR-KNOT $®$ is an automated suture fastener recently proposed for valvular surgery. The COR-KNOT® device is designed to make suture fixation faster and to save operation time ${ }^{2}$. A titanium occluder remotely and automatically secures sutures with a single squeeze of the built-in lever. At the same time the device also trims the excess suture tails ${ }^{2}$.

The COR-KNOT® is a relatively new device developed to replace suture tying with a faster procedure during surgery. Preclinical studies confirmed the safety and security of COR-KNOT® ${ }^{\circledR} \mathrm{use}^{3}$, but there is not enough clinical studies have described the safety of COR-KNOT® as a suitable alternative to conventional hand-tied knots. Most of the initial reports on COR-KNOT® were addressing its potential disadvantages like bioprosthesis leaflet perforation ${ }^{4}$, Paravalvular leakage ${ }^{4}$ and delayed distal embolization ${ }^{5}$. Only a few addressed the potential benefits like time savings in cardioplegic arrest and cardiopulmonary bypass ${ }^{6}$.

Limited number of clinical studies presented the ergonomic advantages COR-KNOR® during minimally invasive cardiac surgery. Here we report a series of cases with the very first use of COR-KNOT $®$ in Bangladesh.

\section{Method:}

Case Selection: The cases were initially selected according to the following: 1) Elective cases for minimally invasive single heart valve surgery, 2) Valvular intervention and candidacy were determined by ESC/EACTS guidelines $^{7}$, 3) All concomitant cardiac surgery patients were excluded from the study 4) All patients was evaluated preoperatively by a Heart team consisting of Cardiac Surgeons, Cardiac Anesthesiologist, Interventional Cardiologist and Cardiac Radiologist team. All information was collected retrospectively from patient's medical record and over phone interview. Therefore, there was a 6 months to one year follow up of each patient. An informed consent was obtained from each patient.

Thansthoracic and Transesophageal Echocardiography: All patients underwent standard preoperative 2D and colour Doppler transthoracic echocardiography (TTE) and all parameters were measured according to American Society of Echocardiography guideline ${ }^{8}$. Routine transesophageal echocardiography was done peroperatively in all cases by using Philips ${ }^{\circledR}$ Affinity 50 ultrasound system with $\mathrm{S} 7-3 \mathrm{t}$ TEE probe in $7-3 \mathrm{MHz}$ frequency by the cardiac anesthesiologist.

CT Scan of Chest: CT scan of thorax, abdomen and pelvis with the upper part of the thigh was done routinely. CT images were acquired in a dual-source 64 slice CT scanner (Siemens ${ }^{\circledR}$ Healthcare, Germany) and evaluated by a radiologist blinded towards the study. CT images were evaluated preoperatively for decision making regarding the surgical approach and femoral vessel assessment for Cardiopulmonary bypass cannulation strategy.

Statistical Analysis: Continuous variables were assessed by Mean \pm Standard Deviation and categorical data were presented as the number and percentage. All analysis was performed using SPSS 16.0.

\section{Technique:}

Five patients had different pathology mostly having rheumatic heart disease. All the mitral valve were approached through a mini left thoracotomy and cannulated via right femoral vessels using $E O P A \circledast$ and multistage Medtronic $₫$ femoral venous cannula. The Aortic valve was approached an upper-J mini-sternotomy and central cannulation technique. Mitral valve cases were ventilated with one lung ventilation (OLV) using double lumen endotracheal tube. The right lung was selectively isolated during the procedure. Cardioplegia was delivered through MiAR ${ }^{\mathrm{TM}}$ aortic root cannula. All patients received DelNido long acting cardioplegia for myocardial protection.

Our first patient has an isolated chordal rupture with prolapsed P2 resulting in severe mitral regurgitation. Triangular resection, artificial chordoplasty with ePTFE 4/0 suture and mitral ring annuloplasty was done successfully. 28mm Physio-Il Mitral annular ring was 
secured with 12 interrupted braided sutures. Out of these 10 were secured with COR-KNOT $®$ device with automated metallic clips (Figure-1) and rest were handtied with knot pusher.

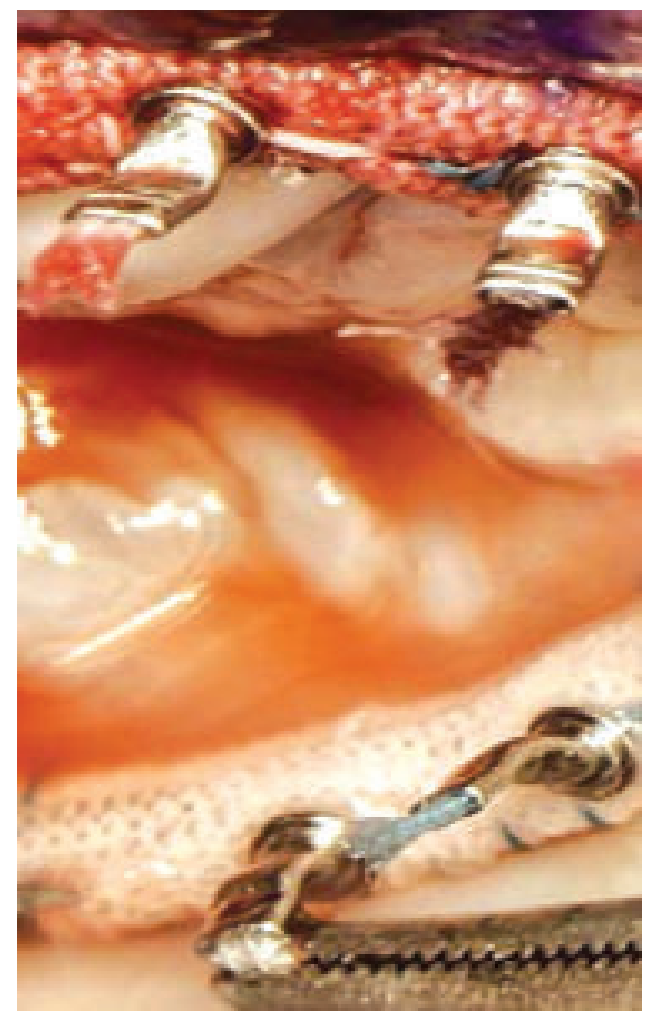

Fig.-1: Automated metallic clips at mitral ring delivers via COR-KNOT® device.

The Second patient had an A2 segment prolapse with rheumatic subvalvular changes. A mitral valve repair was attempted with artificial chordoplasty and mitral ring annuloplasty with a Physio-II ring. Initially, 9 COR-KNOT® were used to secure the ring at mitral annulus. Peropertive Transesophageal Echocardiography showed moderate residual leakage was identified. Re-exploration and COR-KNOT $®$ explantation was done. Mitral valve replacement was carried with a $29 \mathrm{~mm}$ SJM mechanical prosthesis in this young lady. This time 4 COR-KNOT® were used to secure the valve in place and rest other pledgeted stitches were hand-tied by using knot-pusher.

The $3^{\text {rd }}$ case was of severe calcified mixed aortic stenosis and regurgitant. He had a mini sternotomy and Trifecta ${ }^{T M}$ bioprosthetic heart valve replacement. He had 9 COR-KNOT® clips in aortic annulus and rest 3 hand- tied knots at the three aortic commissures. The subsequent case was an elderly lady with severe mitral stenosis due to rheumatic heart disease. We replaced the mitral valve with a SJM Epic ${ }^{T M} 29 \mathrm{~mm}$ stented tissue valve. In this case 12 COR-KNOT® were used to secure the heart valve, rest 4 were hand-tied sutures tied with knot-pusher device.

The last case was a Redo-Mitral valve replacement in a rheumatic mitral re-stenosis patient. This lady had an open mitral commissurotomy done 20years ago. With a similar fashion we approached in this case, replaced the mitral valve with a $29 \mathrm{~mm}$ SJM mechanical prosthesis. Here 10 COR-KNOT® automated clips were used and rest were hand-tied. The biggest hurdle in such case was to mobilize the cardiac apex, which remain stuck with the pericardium and defibrillation via the small thoracotomy. Hence, we recommend the use of external defibrillator pads to be used in such case.

\section{Results:}

First 5 patients with valvular heart disease who underwent valve repair or replacement surgery in a minimally invasive fashion with a Key-hole approach where the knot security were ensured by using automated knotting device - CORKNOT®. First 2 cases were done at National Heart Foundation Hospital \& Research Institute, Dhaka on $3^{\text {rd }}$ December 2015 and subsequent 3 cases were carried out at Ibrahim Cardiac Hospital \& Research Institute, Dhaka on $5^{\text {th }} \& 6^{\text {th }}$ February 2017 . Total 54 shots of metallic automated knots were used in 5 different cases delivered via COR-KNOT® delivery system. Patient demographics and preoperative baseline characteristics are shown in Table-1.

Overall, preoperative CT (Thorax, abdomen and pelvis) showed valvular calcification. Ascending aorta and femoral vessel were also assessed for safe cannulation strategy. Preoperative Transthoracic Echocardiography (TTE) was a part of routine test which was confirmed an on table high resolution Transesophageal Echocardiography (TEE). The result of both the echo was comparable, but the postoperative TEE was much helpful in assessing the repaired heart valve and confirmation of de-aeration of the cardiac chambers.

In all cases a serial documentation of the peri-operative and early postoperative data was done, which is displaced on Table-2 along with the postoperative complications. In this case series there was no use of IABP or any other supporting device. Only one patient developed postoperative new onset atrial fibrillation which didn't require any additional anticoagulation. All 
cases 2 chest tubes were inserted; for mitral valve cases one on the right pleural space and the other used to drain mediastinum. Whereas for the aortic valve case we used both mediastinal drains.

Table-I

Preoperative baseline characteristics

\begin{tabular}{|c|c|}
\hline Preoperative characteristics & $\mathrm{N}=05$ \\
\hline Age (yrs) & $53 \pm 6$ \\
\hline Women (\%) & $3(60)$ \\
\hline Body mass index $(\mathrm{kg} / \mathrm{m} 2)$ & $24.3 \pm 3$ \\
\hline NYHA classiûcation III/IV (\%) & $3(60)$ \\
\hline Diabetes mellitus (\%) & $1(20)$ \\
\hline Hypertension (\%) & $4(80)$ \\
\hline Dyslipidaemia (\%) & $1(20)$ \\
\hline Previous MI (\%) & $0(0)$ \\
\hline Previous cardiac surgery (\%) & $1(20)$ \\
\hline Peripheral artery disease (\%) & $1(20)$ \\
\hline Previous stroke (\%) & $1(20)$ \\
\hline Permanent Pacemaker (\%) & $0(0)$ \\
\hline COPD (moderate/severe) (\%) & $0(0)$ \\
\hline Haemoglobin level (g/dL) & $11.3 \pm 1$ \\
\hline eGFR (mL/min/1.7m2) & $46 \pm 13$ \\
\hline Antiplatelet & $2(40)$ \\
\hline Anticoagulant & $4(80)$ \\
\hline Beta-blocker & $3(60)$ \\
\hline \multicolumn{2}{|c|}{ Transthoracic echocardiography (TTE) } \\
\hline Ejection fraction (\%) & $52.3 \pm 10$ \\
\hline LVIDd (mm) & $59.1 \pm 4.35$ \\
\hline LIVDs (mm) & $48.21 \pm 4.2$ \\
\hline PASP (mm Hg) & $39 \pm 10$ \\
\hline
\end{tabular}

NYHA = New York Heart Association; MI = Myocardial infraction, COPD = Chronic obstructive pulmonary disease; eGFR = estimated glomerular ûltration rate, PASP $=$ Pulmonary systolic artery pressure

All patients were discharged as they improved and there was no wound related complication and no death was reported in 30 days. We reviewed the entire patient in postoperative follow up clinic with a TTE at 1 month, 6 months and one year. There was no paravalvular leak; no treatable pericardial effusion and no prosthesis damage were noted. One patient was readmitted at 3 months follow up due to high INR and discharged after a better titration achieved. One patient in the series died after one and half year due to pregnancy related complication.
Table-II

Perioperative variables and complications

\begin{tabular}{lc}
\hline Peri-operative characteristics & $\mathrm{N}=05$ \\
\hline Cross-clamp time (min) & $78 \pm 9.6$ \\
CPB time (min) & $110 \pm 15.6$ \\
AV block (\%) & $0(0)$ \\
Need for PPM (\%) & $0(0)$ \\
Wound Infection (\%) & $0(0)$ \\
Stroke (\%) & $0(0)$ \\
TIA (\%) & $0(0)$ \\
New onset AF (\%) & $1(20)$ \\
Re-exploration for bleeding (\%) & $1(20)$ \\
Major vascular complication (\%) & $1(20)$ \\
Peri-operative MI (\%) & $0(0)$ \\
Acute Renal Failure (\%) & $0(0)$ \\
Prolong ventilation (>12hr) & $1(20)$ \\
Amount of blood loss (ml) & $180 \pm 32$ \\
Length of ICU stay (day) & $2.1 \pm 0.9$ \\
Length of Hospital Stay (day) & $5 \pm 1$ \\
Readmission for pleural drainage & $0(0)$ \\
Deep Vein Thrombosis & $0(0)$ \\
Mortality (30 days) & $0(0)$ \\
\hline
\end{tabular}

$\mathrm{CPB}=$ Cardiopulmonary Bypass, $\mathrm{AV}=$ Atrio ventricular, $\mathrm{PPM}=$ Permanent pace maker, TIA = Transient Ischemic attack, $\mathrm{AF}=$ Atrial Fibrillation, $\mathrm{MI}=$ Myocardial infraction, $\mathrm{ICU}=$ Intensive Care Unit

\section{Discussion:}

The purpose of this case series to access the clinical characteristics and outcome of automated metallic occluder delivered via COR-KNOT® device. The safety and efficacy of COR-KNOT® was questioned ${ }^{9}$ in some cases of heart valve replacement surgery; especially with the use of bioprosthesis. In our series we didn't experience such complications up to one year postoperative follow up. The present study population although minimum but covered most of the spectrum of heart valve surgery. These cases were selected prospectively to comply with the learning curve and as a part of the preceptorship program lead by a team from National University Hospital, Singapore. And the cases were selected on basis of the incremental difficulty; however the outcome was excellent and suggested the feasibility to introduce new technology in Bangladesh.

The use of COR-KNOT® was proven in Ex-vivo model ${ }^{10}$ in terms of its sutures security, strength, and consistency and it was found faster than manually tied knots while anchored and tested in mitral ring annuloplasty. COR$\mathrm{KNOT} \circledast$ in our cases was clearly able to reproduce the equal outcome compared to hand-tied knots. Some 
more, it exhaled the use of knot-pusher. We made a mix of the use of knot-pusher, hand-tired knot and CORKNOT® in all cases for two reasons. Firstly to keep the attending surgeons to learn the different ways to knot security in Key-Hole heart valve surgery. Secondly, different knot security was clinically evaluated by transthoracic echocardiographic assessment in the same patient at the same time.

However, variable distribution of pathological lesions in our series lead to a different range of surgical accomplishment. It proved the efficacy of COR-KNOT $\circledR$ for heart valve repair, replacement and redo cases. The use of combination of CT scan and preoperativeperoperative-postoperative follow up echocardiography was useful to unravel the clinical outcome of the prosthesis. Nonetheless, this first study in Bangladesh depicts the successful clinical outcome of the CORKNOT $®$ in heterogeneous rheumatic heart valve patients.

Looking at the duration of operation and hospital stay was not more than the conventional cases of heart valve surgery in our study. Candice et al. has shown a clear benefit of titanium fastener use ${ }^{10}$. They reported shorter knotting time, aortic crossclamp time, cardiopulmonary bypass time, shorter duration of operation with fewer intraoperative complications in a randomized clinical trial in open aortic valve replacement.

\section{Conclusions:}

We conclude, COR-KNOT® is a safe and effective tool to reduce the duration of operation. This device is a timesaving secret in heart valve surgeries, especially in minimally invasive settings. Clinical outcome of heart valve surgery with COR-KNOT® is comparable with other methods of suture tying methods.

Acknowledgement: We thank the team from National University Hospital, Singapore headed by Prof. Theodoros Kofidis for their valuable help.

\section{References:}

1. Cushieri A, and Szabo Z; Intracorporeal knots in endoscopic surgery, in Crosthwaite G (Ed): Tissue Approximation in Endoscopic Surgery; Oxford, United Kingdom, Isis Medical Media, 1995, pp 42-48

2. Dan Loberman, Rephael Mohr, Paul Pirundini et al; Automated fastener (Core-Knot) versus manually tied knots in patients undergoing aortic valve replacement Impact on cross-clamp time and short-term echocardiographic results; Medicine (2018) $97: 31$

3. Gersak BM and Sauer JS; In vitro testing of titanium knot fastener used in cardiac and general surgery with pull apart force; J Cardiothorac Surg. 2013; 8(Suppl 1): P64

4. Alexander Brescia, Steven Bolling, and Himanshu Patel et al; Valvular Regurgitation After Implantation of Prostheses Secured With Cor-Knot Automated Fasteners; Ann Thorac Surg 2017;103:e491-2

5. Garrett HE; Delayed Metallic Embolization of a CorKnot Fastener; Thorac Cardiovasc Surg Rep. 2017 Jan; 6(1): e40-e41

6. Martin TR Grapow, Miroslawa Mytsyk, Jens Fassl et. al; Automated fastener versus manually tied knots in minimally invasive mitral valve repair: impact on operation time and short- term results; J Cardiothorac Surg. 2015; 10: 146

7. Guidelines on the management of valvular heart disease (version 2012): The Joint Task Force on the Management of Valvular Heart Disease of the European Society of Cardiology (ESC) and the European Association for Cardio-Thoracic Surgery (EACTS); European Heart Journal, Volume 33, Issue 19, 1 October 2012, Pages 2451-2496

8. Echocardiographic Assessment of Valve Stenosis: EAE/ASE Recommendations for Clinical Practice Helmut Baumgartner et al; Journal of the American Society of Echocardiography; January 2009 Volume 22, Issue 1, Pages 1-23

9. Robert Balan, Christian Mogilansky, Axel-Lars Larsen et. Al; Severe aortic regurgitation after implantation of a sutureless valve prosthesis using an automatic knot fastener device; Interactive CardioVascular and Thoracic Surgery, Volume 25, Issue 1, 1 July 2017, Pages 153-154

10. Candice Y. Lee, Jude S. Sauer, Heather R. Gorea et. Al; Comparison of Strength, Consistency, and Speed of COR-KNOT Versus Manually Hand-Tied Knots in an Ex Vivo Minimally Invasive Model; Innovations \& Volume 9, Number 2, March/April 2014:111-116 